RESEARCH PAPER RP676

Part of Bureau of Standards Journal of Research, vol. 12, May 1934

\title{
FREEZING POINT OF RHODIUM
}

\author{
By Wm. F. Roeser and H. T. Wensel
}

\section{ABSTRACT}

The freezing point of rhodium was determined by measuring with a disappearing filament type of optical pyrometer the ratio of brightness of black bodies immersed in freezing gold and freezing rhodium. The rhodium was melted and frozen in vacuo to prevent the absorption of gases during melting. Attempts to operate with the rhodium in atmospheres of air and of nitrogen were not successful.

The ratio of brightness of black bodies at the freezing points of gold and rhodium was found to be 751 for wave length $0.6527 \mu$. This gives 1,966 degrees centigrade for the temperature of freezing rhodium when the gold point is fixed at $1,063.0 \mathrm{C}$ and $\mathrm{C}_{2}$ at $1.432 \mathrm{~cm}$-degrees. The value 1,966 degrees centigrade is probably accurate to $\pm 3^{\circ} \mathrm{C}$.

CONTENTS

Page

I. Introduction _.

II. Previous work

III. Present work

IV. Results__._.

V. Preparation and purity of the rhodium

\section{INTRODUCTION}

The development of a practical form of hollow inclosure or black body immersed in freezing platinum ${ }^{1}$ and the demonstration that the radiation from this form of black body is sufficiently constant and reproducible to serve as a standard of light, immediately suggested the possibility of employing metals with higher melting points for this and similar purposes. The metals of the platinum group appear to be the most suitable for this purpose. The investigation of black bodies immersed in freezing rhodium and iridium was made primarily to determine the advantages and disadvantages of these metals for establishing standards of luminous intensity.

One application of this form of immersed black body has been the establishment of a scale of color temperature. This work, described elsewhere ${ }^{2}$ in this Journal, required an accurate knowledge of the freezing points of at least three metals. The three selected, platinum, rhodium, and iridium, melt at temperatures which are well spaced on the scale of chromaticity.

\section{PREVIOUS WORK}

Previous observations on the melting point of rhodium are summarized and reduced to the International Temperature Scale ${ }^{3}$ in table 1. In two cases, the apparent or brightness temperature of the

1 B.S.Jour. Research, vol. 6, p. 1103, 1931.

2 B.S.Jour. of Research, vol. 12 (RP 677), p. 527,1934

3 Proc. 7th Gen. Conf. Weights and Measures, p. 56, 1927; Text in annex 4, p. 94; B.S.Jour. Research, vol. 1 , p. 635,1928 . 
metal at its melting point was reported. In deriving values for the true temperature from these, the value 0.29 , reported by Henning ${ }^{4}$ and also by Burgess and Waltenberg, ${ }^{5}$ was used for the emissivity. All of the previous determinations were made by methods differing either in principle or detail and little can be said regarding their relative accuracy with the exception that Henning and Heuse improved upon the method used by Holborn and Henning and obtained a value approximately $70^{\circ} \mathrm{C}$ higher. The tentative value reported by Swanger was preliminary to the present work.

TABLE 1.-Summary of determinations of the melting point of rhodium byvarious observers

\begin{tabular}{|c|c|c|c|c|c|c|c|c|}
\hline \multirow{3}{*}{ Observer } & \multirow{3}{*}{ Year } & \multicolumn{3}{|c|}{ Scale used } & \multicolumn{3}{|c|}{ Reported values } & \multirow{3}{*}{\begin{tabular}{|c} 
True \\
tempera- \\
ture on \\
Inter- \\
national \\
Temper- \\
ature \\
Scale
\end{tabular}} \\
\hline & & \multirow{2}{*}{$\underset{\text { point }}{\mathrm{Au}}$} & \multirow{2}{*}{$\underset{\text { point }}{\mathrm{Pt}}$} & \multirow{2}{*}{$\mathrm{C}_{2}$} & \multicolumn{2}{|c|}{ Temperature } & \multirow{2}{*}{$\begin{array}{l}\text { Wave- } \\
\text { length }\end{array}$} & \\
\hline & & & & & Apparent & True & & \\
\hline Holborn and $\mathrm{H}$ & \multirow{5}{*}{$\begin{array}{l}1905 \\
1907 \\
1910 \\
1916 \\
1924 \\
1929 \\
1933\end{array}$} & $\begin{array}{l}{ }^{\circ} \mathrm{C} \\
1,064\end{array}$ & ${ }^{\circ} \mathrm{C}$ & \multirow[t]{2}{*}{1.45} & $\begin{array}{l}{ }^{\circ}{ }_{1,650} \\
\text {. }\end{array}$ & ${ }^{\circ} \mathrm{C}$ & \multirow[t]{2}{*}{$\begin{array}{l}\mu \\
0.643\end{array}$} & \multirow{5}{*}{$\begin{array}{l}{ }^{\circ} \mathrm{C} \\
1,891 \\
1,946 \\
1,980 \\
1,974 \\
1,958 \\
1,984 \\
1,966\end{array}$} \\
\hline Mendenhall and Inge & & 1,065 & $\left.\begin{array}{l}1,745 \\
1,789\end{array}\right\}$ & & & $\left\{\begin{array}{l}1,907 \\
1,968\end{array}\right\}$ & & \\
\hline $\begin{array}{l}\text { Von Wartenberg }{ }^{3}- \\
\text { Burgess }\end{array}$ & & & $\begin{array}{l}1,745 \\
1,7--\end{array}$ & \multirow{3}{*}{$\begin{array}{l}1.46 \\
1.445 \\
1.43 \\
1.432 \\
1.432\end{array}$} & & $\begin{array}{l}1,940 \\
1,960\end{array}$ & \multirow{3}{*}{0.622} & \\
\hline ind Heyse & & 1,063 & -0 & & 1,720 & & & \\
\hline Present wor & & $\begin{array}{l}1,063 \\
1,063\end{array}$ & $\ldots$ & & & $\begin{array}{l}1,984 \\
1,966\end{array}$ & & \\
\hline
\end{tabular}

1 Preuss. Akad. Wiss. Berlin, Sitz. Ber., vol. 12, p. 311, 1905.

2 Phys. Rev., vol. 25, p. 1, 1907.

3 Verh. Deut. Phys. Gesell., vol. 12, p. 121, 1910.

4 Jour. Frank. Inst., vol. 182, p. 19, 1916.

5 Zeits. f. Physik., vol. 29, p. 157, 1924.

6 B.S.Jour. Research, vol. 3, p. 1029, 1929.

In all of these determinations, with the exception of the two reported by Von Wartenberg and by Swanger, an optical pyrometer was sighted either on samples of rhodium heated electrically in air or on small bits of the metal placed on electrically heated materials. Von Wartenberg melted small samples of rhodium in a tungsten tube in vacuo. In the work reported by Swanger 5 to $10 \mathrm{~cm}^{3}$ of the metal was heated in vacuo in a high-frequency induction furnace and an optical pyrometer was sighted either into a hole drilled into the metal or into an immersed sight tube similar to that used in the present work.

\section{PRESENT WORK}

In view of the applications to be made of the freezing point of rhodium, it was considered advisable to use the black body under exactly the same conditions for the temperature measurements, the brightness measurements, and the color comparisons. The last two require the use of a lens to form a magnified image of the opening in the black body, and the small size of this opening required that the distance between it and the lens should not exceed $17 \mathrm{~cm}$ to secure sufficient magnification to fill the field of the photometers available. This, of course, limits the distance between the observation window and the rhodium if the metal is to be melted in an atmosphere other than air.

One of the sources of error in the determination of the brightness of a black body by the method described in the paper on the Waidner-

Zeits. f. Instrumentenkunde, vol. 30, p. 61, 1910.

5 B.S. Bulletin vol. 11 (SP242), p. 591, 1915. 
Burgess Standard of Light, ${ }^{6}$ arises from the uncertainty in the value of the transmission of the lens and prism. The use of a black body immersed in freezing rhodium in an atmosphere other than air, requires the determination of the transmission of a window (which could seldom be kept entirely free from a film) in addition to that of the lens and prism. The film could have been avoided by using a longer tube as described by Swanger but it would not have been possible to make brightness measurements or color temperature comparisons under these conditions. In order to avoid the additional uncertainty due to the film on the window, a futile attempt was made to operate with the rhodium in air. It was known in advance that rhodium when melted in air absorbs gas which, during freezing, is released rather violently with the formation of "sprouts." The rhodium was mounted in a thorium oxide crucible in the same manner as in the case of platinum ${ }^{7}$ and iridium. ${ }^{8}$ In spite of the fact that the cover was tightly sealed on the crucible and that the metal was maintained above its melting point for only a few minutes, it sprouted severely, breaking the sight tube on the second freeze. Some of the sprouts through the cracks in the crucible extended as far as $2 \mathrm{~cm}$ into the insulation.

Attention was then directed toward operating with the rhodium in vacuo. The metal and crucible ${ }^{9}$ were packed in a porcelain container which fitted into a pyrex tube about $7 \mathrm{~cm}$ in diameter. The pyrex tube was about $25 \mathrm{~cm}$ long and was provided with a tube for evacuating. The opening in the black body was about $16 \mathrm{~cm}$ from the pyrex window used in sealing the tube. Considerable difficulty was experienced with the film collecting on the inside of the window. Chemical and optical tests made to identify the material collecting on the window indicated that it was thorium oxide. In the first experiments the film collected on the window at a rate which produced a drift of about $1^{\circ} \mathrm{C}$ per freeze. In later experiments 3 diaphragms were placed between the window and the rhodium packed in the thoriun oxide. The metal was maintained at a temperature just below its melting point for a total of 12 hours before making observations. After the window was cleaned the observation showed a drift of about $0.1^{\circ} \mathrm{C}$ per freeze. Examples of groups of observations with a large and a small drift are given in figure 1. In other experiments a fused silica window was placed inside the evacuated tube and connected to an iron rod in a side tube, so that the window could be placed over the opening in one of the diaphragms by means of a magnet during melting and moved aside during freezing. The observations then indicated a drift of about $0.3^{\circ} \mathrm{C}$ per freeze. The difference between the amount of film collected on the window in these experiments and in the preceding set of experiments is probably due to the fact that the insulation was not heated for an appreciable length of time before taking the observations.

In some of the above experiments, the effect of the final film on the window was also determined by measuring the transmission (for red light) of the window before and after a series of observations at the rhodium point. The change in the transmission of the window obtained in this way agreed with that indicated by the drift in the

\footnotetext{
${ }_{6}^{6}$ B.S.Jour. Research, vol. 6, p. 1103, 1931.

$?$ B.S.Jour. Research, vol. 6, p. 1119, 1931.

8 B.S.Jour. Research,

Acknowledgment is made to F. R. Caldwell who prepared the crucibles and black bodies and assisted in the observations.
} 
observations. The pressure during the observations was between 0.001 and $0.05 \mathrm{~mm}$ of $\mathrm{Hg}$. A liquid-air trap was placed between the diffusion pump and the tube containing the rhodium to prevent mercury vapor from diffusing back into this tube.

An attempt to melt and freeze the rhodium in an atmosphere of nitrogen was not successiul. The sight tube broke during the.second freeze. The film collected on the window in this case at about the same rate as in vacuo and it therefore was concluded that little could

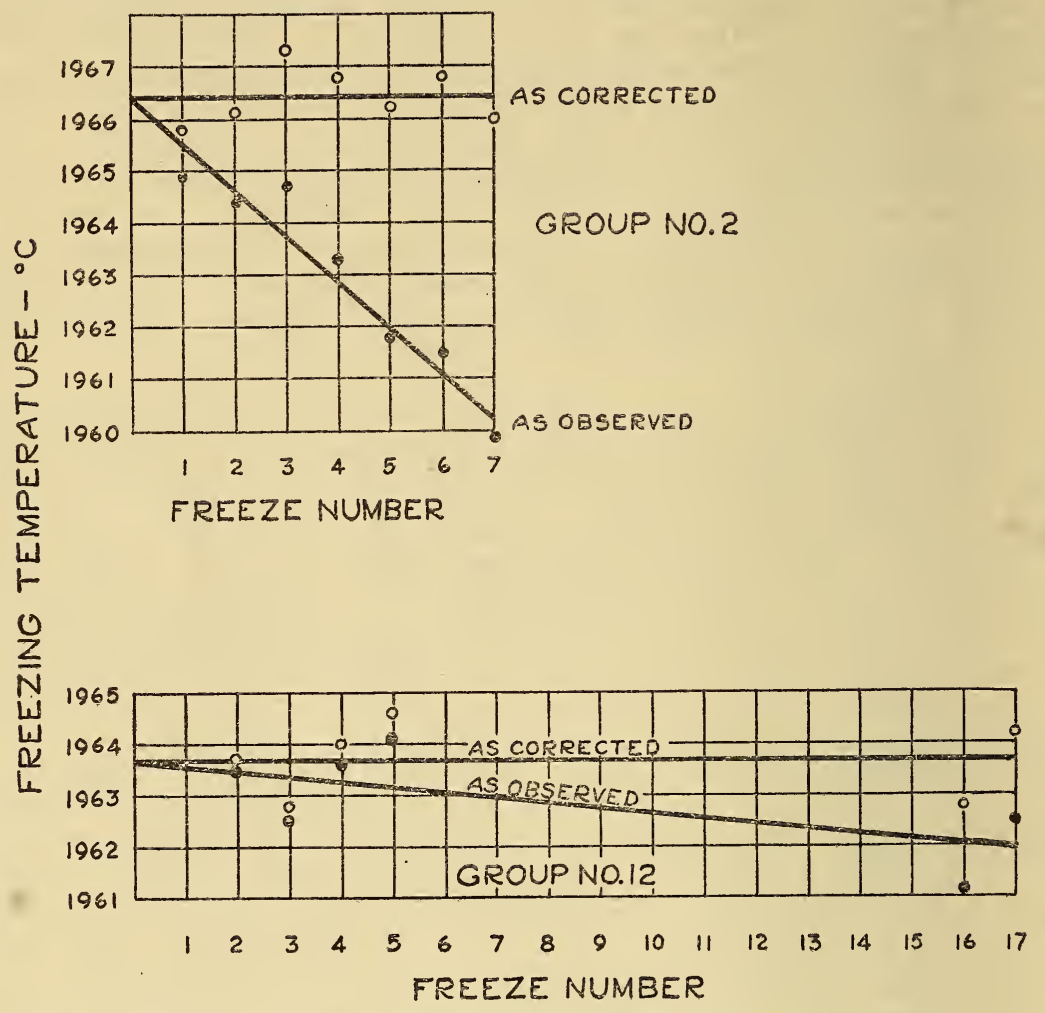

FIgURE 1.-Examples of the drift in the observations at the freezing point of rhodium due to film collecting on the window.

be gained by operating with the rhodium in an atmosphere of nitrogen.

The method of observation and the pyrometer were essentially the same as those used in determining the freezing points of platinum and iridium. Since the ratio of brightness of black bodies at the gold and rhodium points is approximately 750 for red light, a sector disk with less than 30 minutes of arc opening would be required if the ratio were to be measured in one step. Inasmuch as an opening of this size could not be measured with sufficient accuracy, the ratio of brightness was measured in two steps using two different combinations of sector disks. In each case, a black body was operated at a temperature between the freezing points of gold and rhodium. The intermediate temperature was $1,220 \mathrm{C}$ in one case and $1,360 \mathrm{C}$ in the other. The ratio of brightness of black bodies at the platinum and rhodium points was also measured. This ratio of brightness is 
approximately 2.5 , which requires a sector disk with about 40 percent transmission.

Observations at the freezing points of platinum and gold taken before and after the observations at the rhodium point showed no change in the pyrometer lamp during the course of this work.

The mean efiective wave length of the red glass filter for the individual observers participating in this work was determined from the comparisons reported in the paper on "The Freezing Point of Iridium." The temperatures were calculated as described in the paper on "The Freezing Point of Platinum", with the exception that it was necessary to correct for the film collecting on the window.

In those cases in which a number of freezes were taken and the drift in the observations indicated that the correction was directly proportional to the number of freezes, correction was made on that basis. In other cases where only one or two freezes were taken and it was not possible to establish a drift in the observation, the change in the transmission of the window (for red light) was used to determine the correction to be applied.

\section{RESULTS}

The calculated results are given in table 2 . With the exceptions of groups 7,11 , and 13, the window was cleaned before each group of observations: After the observations in group 4, six freezes were used for another purpose and then the observations in group 11 were taken; all the observations in group 7 were taken between the 4 th and 5 th observation given in group 12; the observations in group 13 immediately followed those in group 9.

The ratio of brightness of black bodies at the freezing points of gold and rhodium was found to be 751 at wave length $0.6527 \mu$ from which the temperature of freezing rhodium was calculated as 1,966 degrees centigrade on the international temperature scale. At the same wave length the ratio of brightness of black bodies at the freezing points of platinum and rhodium was found to be 2.52 . This ratio is consistent with the values $1,773.2$ and 1,966 degrees centigrade for the freezing points of platinum and rhodium, respectively, to within about $0.3^{\circ} \mathrm{C}$.

It is estimated that the error in the final result due to uncertainty in the transmission of the sector disks, effective wave length, photometric matching, departure from black body conditions and impurities in the metal, does not exceed $2.5^{\circ} \mathrm{C}$ if all are given the maximum value and if all are of the same sign. Uncertainty in any of the first three sources may cause the final result to be either high or low whereas that due to the departure from black body conditions would result in a low value for the freezing point.

The difierence $\left(1.7^{\circ} \mathrm{C}\right)$ between the mean of groups 3 to 10 and that of groups 11 to 13 would seem to indicate that the uncertainty in the measurement of the transmission of the sector disks is greater than that estimated. However, part of this difference may be due to the uncertainty in the correction applied for film on the window. Most of the observations in groups 11 to 13 were taken after some of those in groups 3 to 10 , and required the application of larger corrections because the window was not cleaned in the meantime. The results do not show any systematic error in the method used in 
applying the correction for the film. The final result, 1,966 degrees Centigrade for the freezing temperature of rhodium is probably accurate to $\pm 3^{\circ} \mathrm{C}$.

TABLE 2.-Summary of observations at the freezing point of rhodium

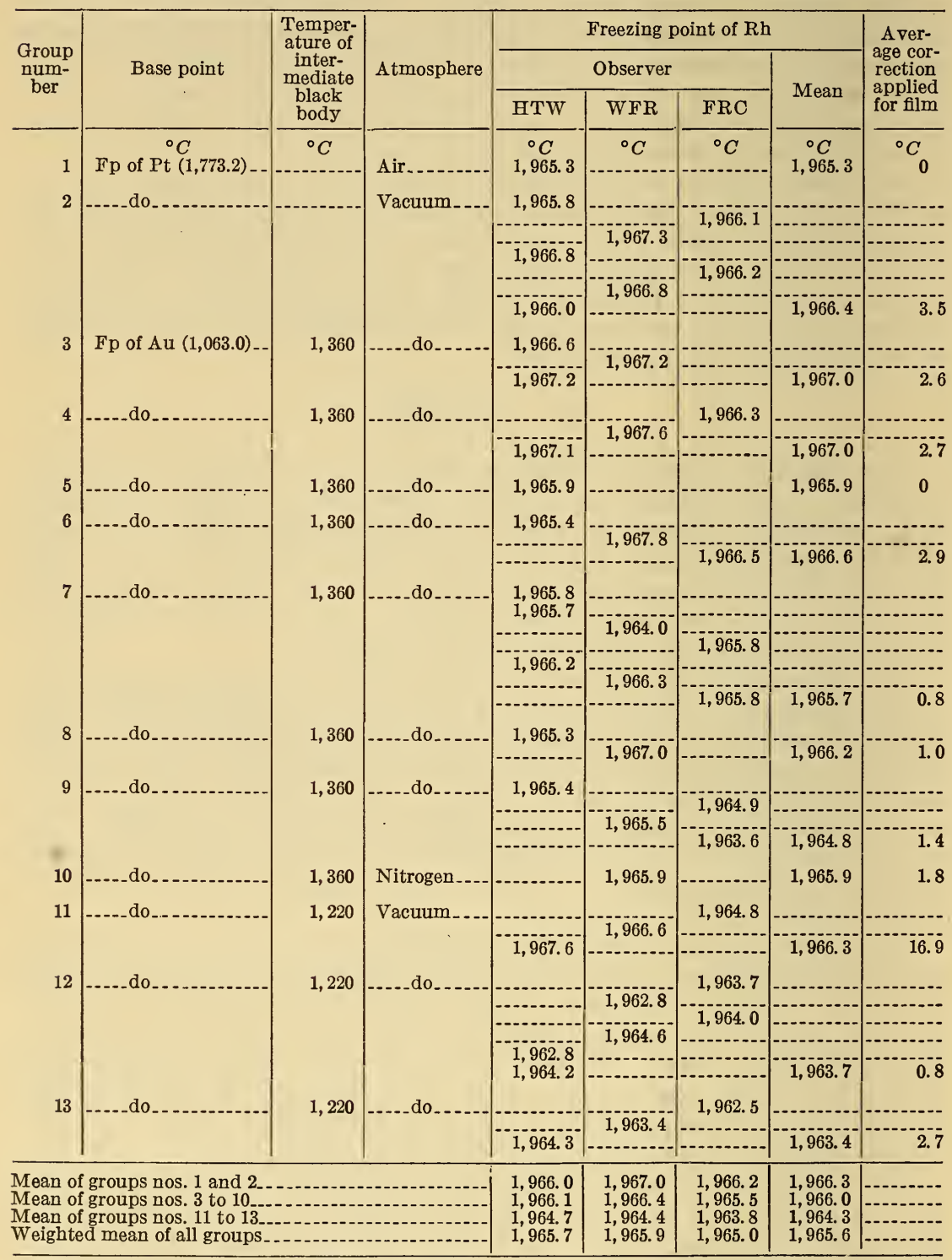

\section{[FREEZING POINT OF RHODIUM, 1,966 DEGREES CENTIGRADE]}

In view of the fact that the film collecting on the window produced an uncertainty in the determination of its light absorption, the black body at the freezing point of rhodium, as set up in this work, is not considered suitable for use as a standard of luminous intensity. 


\section{PREPARATION AND PURITY OF THE RHODIUM}

\section{By Edward Wichers}

The rhodium used in this work was carefully purified ${ }^{10}$ by a procedure which corresponded for the most part with that described by Wichers, Gilehrist, and Swanger. ${ }^{11}$ Since the publication of that paper, further experience has shown that the repeated precipitation of ammonium rhodium nitrite, although very effective for removing other impurities, is not satisfactory for removing more than very small amounts of iridium. In order to reduce the iridium content of the rhodium to an amount suitable for the application of the nitrite process, the following method was used for effecting solution of the rhodium. The impure rhodium was mixed with about 20 parts of lead and the mixture fused in a graphite crucible at about 1,000 $\mathrm{C}$ for half an hour. ${ }^{12}$ The resulting alloy was treated with nitric acid to dissolve most of the lead. In this treatment a little rhodium dissolves, but most of it is left as a finely divided alloy with lead. This alloy was vigorously attacked, first with concentrated hydrochloric acid, and then with aqua regia, leaving finally a residue which contained nearly all of the iridium, together with a small portion of the rhodium.

Most of the lead was precipitated from the acid solutions with sulphuric acid, added in slight excess. The filtrates from the lead sulphate were combined and evaporated to a small volume to expel most of the free acids and to obtain a second precipitate of lead sulphate. The filtrate from this precipitate was treated with a sufficient amount of a solution of sodium nitrite to neutralize the remaining free acid and to convert the rhodium chloride into sodium rhodium nitrite, $\mathrm{Na}_{3} \mathrm{Rh}\left(\mathrm{NO}_{2}\right)_{6}$. The solution was boiled to complete the conversion to nitrite. To this solution, which contained about $50 \mathrm{~g}$ of rhodium per liter, was added about $30 \mathrm{~g}$ of sodium sulphide crystals for each $100 \mathrm{~g}$ of rhodium. This was done to precipitate certain base metal impurities, such as copper and lead. Some of the platinum metal impurities, especially palladium, are partially precipitated by this treatment, but very little of the rhodium is lost. After standing overnight, the sulphide precipitate was filtered off and the solution boiled to consume any remaining sodium sulphide by its reaction with platinum metal impurities or with the rhodium itself. After removing the second small precipitate of sulphides, the solution was cooled to about $15 \mathrm{C}$ and treated with a solution of ammonium chloride to precipitate a salt which has generally been regarded as $\left(\mathrm{NH}_{4}\right)_{3} \mathrm{Rh}\left(\mathrm{NO}_{2}\right)_{6}$ but which may be a mixed sodium and ammonium rhodium nitrite. About $200 \mathrm{~g}$ of $\mathrm{NH}_{4} \mathrm{Cl}$ was used for each $100 \mathrm{~g}$ of rhodium. This proportion includes a small excess of ammonium chloride, which decreases the solubility of the double salt.

After filtering and washing the salt, it was decomposed with hydrochloric acid diluted with 3 or 4 volumes of water. The chloride solution was then evaporated to expel most of the hydrochloric acid and the cycle of operations with sodium nitrite, sodium sulphide, and ammonium chloride repeated six times. The quantity of sodium sulphide used was decreased each time, and the sulphide precipitates were examined chemically for lead. Lead was not detected after the

\footnotetext{
10 By R. K. Bell.

11 "Purification of the Six Platinum Metals", Trans. Am. Inst. Mining Met. Eng., vol. 76, p. 602, 1928. 12 Although it might sometimes be disadvantageous to add lead to the rhodium, because of the difficulty of eliminating all of that metal in the subsequent process of purification, it is usually of no consequence, because lead is very often already present as an impurity in the rhodium.
} 
fourth precipitation. At this stage a solution of sodium nitrite which had been treated to remove traces of base metals was substituted for the ordinary reagent.

Spectrographic examination of the rhodium from the fifth precipitation failed to disclose the presence of any other platinum metals, but indicated the presence of a trace of lead. Lead was not found in the metal from the sixth precipitation. The salt obtained by the seventh precipitation was converted into a chloride solution, by means of hydrochloric acid, and ammonium chloride was added in slight excess of the amount equivalent to $\left(\mathrm{NH}_{4}\right)_{3} \mathrm{RhCl}_{8}$. The double chloride was then precipitated, from this solution, containing about $100 \mathrm{~g}$ of rhodium per liter, by adding one and one half times its volume of 95 percent alcohol. The salt was washed with alcohol, dissolved in water, and reprecipitated by means of alcohol from a somewhat more dilute solution. The salt thus prepared contained, after drying, 30.7 percent of rhodium, which is about midway between the rhodium content of $\left(\mathrm{NH}_{4}\right)_{2} \mathrm{RhCl}_{5}$ and $\left(\mathrm{NH}_{4}\right)_{3} \mathrm{RhCl}_{6}$.

The salt was ignited in a porcelain container in a resistance furnace in such a way as to prevent contamination with metal vapors from the winding. The residue was reduced to rhodium sponge by ignition in hydrogen and the sponge digested with water to extract a small amount of alkali salts. The sponge was then compressed into pellets in a steel mold and melted in a thorium oxide crucible, in vacuo, to form an ingot of the proper shape and size.

The chemicals used in the purification were all of analytical reagent quality, except that some of the sodium nitrite used was specially treated to remove base metals, as previously mentioned. A more detailed discussion of the process of purification is given in the reference cited..$^{13}$

After the work on the determination of the melting point had been completed a portion of the ingot was prepared for spectrographic examination and another for determination of the electrical resistivity and the temperature coefficient of resistance. ${ }^{14}$ The latter specimen was obtained by sawing a quarter segment out of the ingot and workit into wire by the method described by Swanger. ${ }^{15}$

The resistivity was 4.3 microhm-cm at $0 \mathrm{C}$, and the ratio $\frac{\mathrm{R}_{100}-\mathrm{R}_{0}}{100 \mathrm{R}_{0}}$ was 0.00457 . The former value is lower, and the latter higher, than any corresponding value found in the literature, thus indicating that this rhodium had a higher degree of purity than any whose electrical properties have been reported heretofore. Spectrographic examination ${ }^{16}$ gave no evidence that the rhodium had taken up thorium from the refractory crucible or had been otherwise contaminated during the experimental work, except perhaps by a trace of iron, presumably introduced during the forging of the metal or the drilling of the ingot for the sight tube, in spite of the fact that the ingot was digested with boiling hydrochloric acid after each such treatment. It is believed that the total metallic impurities in the rhodium, at the completion of the work, did not exceed 0.01 percent, and may have been considerably less.

Washington, March 1, 1934.

\footnotetext{
13 See footnote 11 .

14 Both determinations made by Wm. F. Roeser.
}

$1 s$ Melting, Mechanical Working, and Some Physical Properties of Rhodium, B. S. Journal Research, vol. 3, p. 1029, 1929. The specimen was prepared by J. G. Thompson.

10 By B. F. Scribner. 